\title{
Efecto de la serpentina en la formación de fases mineralógicas y su relación con la resistencia mecánica en sinterizados de mineral de hierro ${ }^{(\bullet)}$
}

\author{
E. Bedolla ${ }^{(*)}$, C.A. León ${ }^{(*)}$, J. Lemus ${ }^{(*)}$, E.A. $\operatorname{Aguilar}^{(*)}$ y G. Mendoza ${ }^{(* *)}$
}

Resumen Se estudia la influencia de las microestructuras formadas sobre la resistencia mecánica de mineral de hierro aglomerado con serpentina. Se deduce que la microestructura formada depende de la temperatura de sinterización, de la basicidad y del valor porcentual de serpentina, principalmente. Las fases mineralógicas presentes se cuantificaron utilizando la técnica de conteo por puntos. En briquetas ácidas $\left(\mathrm{CaO} / \mathrm{SiO}_{2}=0,3\right)$ sinterizadas a $1.275,1.300$ y $1.325^{\circ} \mathrm{C}$, se encontró que la serpentina induce la formación de magnesio-ferritas que traen, como consecuencia, una disminución de la resistencia mecánica, mientras que, a la temperatura de sinterización de $1.250^{\circ} \mathrm{C}$, la aparición de escorias vítreas es la causa principal de la disminución de resistencia. En el caso de briquetas básicas $\left(\mathrm{CaO} / \mathrm{SiO}_{2}=1,2\right)$, la adición de serpentina produce la formación de olivinas y calcio-ferritas que originan un aumento de la resistencia mecánica de los sinterizados.

Palabras clave: Mineral de hierro. Serpentina. Sinterizados. Fases mineralógicas. Resistencia mecánica.

\section{Effect of the serpentine on the formation of mineralogical phases and its relation with the mechanical strength in iron ore sinters}

\begin{abstract}
The influence of microstructures formed by serpentine addition on mechanical strength is investigated. It was found that the microstructure formed depends mainly on firing temperature, basicity and serpentine percent. The mineralogical phases present were quantified by the point-count method. The addition of serpentine on acid briquettes $\left(\mathrm{CaO} / \mathrm{SiO}_{2}=0.3\right)$, mainly on those fired at $1,275,1,300$ and $1,325^{\circ} \mathrm{C}$, leads to the formation of magnesio-ferrite that diminishes the mechanical strength of fired briquettes, and at the temperature of $1,250^{\circ} \mathrm{C}$ the mechanical strength decreases because of the presence of glassy slags. On the other hand, the addition of serpentine on basic briquettes $\left(\mathrm{CaO} / \mathrm{SiO}_{2}=1.2\right)$ leads to the formation of olivines and ferrites that improve the mechanical strength of fired briquettes.
\end{abstract}

Keywords: Iron ore. Serpentine. Sintering. Mineral phases. Mechanical strength.

\section{INTRODUCCIÓN}

La completa determinación de la microestructura de los aglomerados de mineral de hierro es una

$(\bullet \quad$ Trabajo recibido el día 3 de mayo de 1995.

(*) Instituto de Investigaciones Metalúrgicas. Universidad Michoacana de San Nicolás de Hidalgo. Apdo. Postal 52-B. Morelia, Mich. (México).

(**) Dpt. of Engineering Materials. The University of Sheffield. Mappin Street. Sheffield S1 4DU (Reino Unido). necesidad básica y el primer paso hacia el establecimiento de una relación estructura-propiedades.

Las propiedades físicas y químicas de los aglomerados de mineral de hierro dependen de la cantidad y de la distribución de las fases presentes, así como de las características de los componentes individuales (tamaño, morfología, tipos de uniones e interacción entre los mismos) que caracterizan a la microestructura y están relacionados directamente con propiedades tales como resistencia mecánica y reducción a baja temperatura (1-3).

La calidad de los aglomerados de hierro se ve afectada en gran parte por la microestructura 
formada durante el proceso de endurecimiento o sinterización, de aquí la importancia de que se prefiera la formación de determinadas fases que proporcionen las características deseadas para obtener un pelet de buena calidad. Uno de los parámetros comúnmente empleados para evaluar la calidad de los sinterizados es la resistencia mecánica en frío (4), cuya técnica de determinación se aplica en el presente trabajo.

A través del desarrollo del proceso de peletización, los aditivos se han usado para mejorar la operación y economía del proceso, por lo que se espera que cumplan los objetivos de mejora de la resistencia de los pelets antes y después del secado, reducción de la temperatura de sinterización del pelet sin detrimento de su calidad y mejora de las propiedades físicas y químicas de los pelets sinterizados (57). Actualmente, existe la tendencia a usar como aditivos fuentes de $\mathrm{CaO}$ y $\mathrm{MgO}$, debido a que promueven la formación de microestructuras tales como la calcio-ferrita y la magnesio-ferrita que aportan buenas propiedades a los aglomerados ya sea en el proceso de endurecimiento o en el de reducción.

Diversos autores (8-12) señalan que las adiciones de $\mathrm{MgO}$ a minerales magnetíticos o hematíticos contribuyen a mejorar las propiedades a alta temperatura de los pelets y aumentan la reducibilidad; a su vez, mayores contenidos de $\mathrm{MgO}$ contribuyen a incrementar la porosidad de los pelets.

La serpentina (silicato de magnesio) es un aditivo que contiene óxidos de magnesio y de silicio, que ha sido motivo de estudio para conocer las propiedades que induce en los aglomerados de mineral de hierro durante los diferentes procesos, tema de estudio que ha sido citado con anterioridad por Bedolla y col. (13).

S.C. Panigrahy y col. (14) investigaron el efecto del $\mathrm{MgO}$ sobre las características de la resistencia mecánica de aglomerados de mineral de hierro usando hematites en un rango de basicidad de 0,7 a 1,9. Los índices de basicidad de los aglomerados los obtuvieron al agregar fundentes tales como caliza, dolomita y dunita, incrementando la relación $\mathrm{MgO} / \mathrm{CaO}$ de 0 a 1,2 con intervalos de 0,2. De acuerdo con sus resultados, se propuso que la resistencia mecánica de los sinterizados depende, en gran medida, de las propiedades y de las cantidades de la matriz resultante, definida como la suma de fase vítrea, silicatos, olivinas y calcio-ferritas.

Dedujeron, además, que el incremento de la relación $\mathrm{MgO} / \mathrm{CaO}$ contribuye a reducir la resistencia mecánica en frío de los sinterizados. Sin embargo, para aglomerados en el intervalo de baja basici$\mathrm{dad}$, pequeñas adiciones de $\mathrm{MgO}$ tienen un efecto beneficioso. Para sinterizados ricos en $\mathrm{CaO}$, la presencia de ferritas es el principal factor que contribuye a la resistencia, y con respecto a sinterizados ricos en $\mathrm{MgO}$, la precipitación de olivinas en la matriz vítrea contribuye a la obtención de la resistencia más elevada. La presencia de gran cantidad de escoria y la precipitación de dicalcio-silicatos parecen tener un efecto adverso sobre la resistencia mecánica de los sinterizados.

La escoria vítrea tiene un alto grado de tensiones atribuido a una diferencia en el coeficiente térmico expansión/contracción entre la fase vítrea y la fase cristalina que encierra. Cuando aparecen como un producto de desvitrificación del vidrio, los dicalciosilicatos son perjudiciales para la resistencia del sínter debido a que a $697{ }^{\circ} \mathrm{C}$, sufren una transformación alotrópica de $\beta-2 \mathrm{CaO} \cdot \mathrm{SiO}_{2}$ a $\gamma-2 \mathrm{CaO}$. $\mathrm{SiO}_{2}$ acompañada por un cambio de volumen y ocasiona la pulverización del sínter.

En los últimos años, los aditivos constituidos por fuentes de $\mathrm{MgO}$ han cobrado relativa importancia debido, fundamentalmente, al comportamiento de los pelets durante el proceso de reducción; es decir, que presentan buena resistencia a la degradación y bajo reblandecimiento, comportamiento que no presentan los pelets que contienen $\mathrm{CaO}$ como fundente principal. Uno de los aditivos fuente de $\mathrm{MgO}$ más ampliamente utilizado es la dolomita, aunque, sin embargo, existen otros, como la serpentina, que lo contienen igualmente y se están empleando debido a los buenos resultados obtenidos.

El presente estudio se realizó con el propósito de evaluar el efecto de la composición, específicamente el nivel de $\mathrm{MgO}$ y la relación $\mathrm{CaO} / \mathrm{SiO}_{2}$, además de la temperatura de sinterización en la formación de fases y su relación con la resistencia mecánica en probetas de mineral de hierro.

\section{PROCEDIMIENTO EXPERIMENTAL}

\subsection{Materiales}

En el presente estudio se utilizó mineral magnético de la empresa minera Las Truchas S.A. Michoacán (México), cuya composición química se muestra en la tabla I, donde se presenta también la composición de los aditivos empleados, serpentina y cal.

TABla I.- Composición química del mineral de hierro y de los aditivos

TABLE I.- Chemical composition of iron ore and additives

\begin{tabular}{|l|ccrrrrc|}
\hline Material & $\mathrm{Fe}_{\mathrm{T}}$ & $\mathrm{Fe}^{2+}$ & $\mathrm{SiO}_{2}$ & $\mathrm{CaO}$ & $\mathrm{MgO}$ & $\mathrm{Al}_{2} \mathrm{O}_{3}$ & $\mathrm{~S}$ \\
\hline $\mathrm{Fe}_{3} \mathrm{O}_{4}$ & 65,59 & 22,512 & 3,21 & 1,47 & 0,38 & 0,75 & 0,0108 \\
Serpent. & - & - & 40,00 & 0,53 & 38,60 & 1,50 & - \\
$\mathrm{CaO}$ & - & - & - & 99,00 & - & - & - \\
\hline
\end{tabular}

Rev. Metal. Madrid, 31 (6), 1995 


\subsection{Preparación de muestras}

El aglomerado utilizado en este estudio se preparó mezclando el concentrado del mineral con 0 , $1,2,3,4$ y $5 \%$ de serpentina, fabricando dos tipos de aglomerados: uno básico $\left(\mathrm{CaO} / \mathrm{SiO}_{2}=1,2\right)$ y otro ácido $\left(\mathrm{CaO} / \mathrm{SiO}_{2}=0,3\right)$, ajustando la basicidad binaria con $\mathrm{CaO}$ de grado reactivo. El balance de materiales de las mezclas empleadas se muestra en la tabla II.

Se fabricaron probetas utilizando la técnica empleada por Gupta y col. (8). Para ello, el mineral de hierro se mezcló con la serpentina y con el óxido de calcio previsto, introduciendo en cada pastilla 0,8 $\mathrm{g}$ de la mezcla en un recipiente cilíndrico cerrado y ejerciendo una presión de 19,6 MPa $\left(200 \mathrm{~kg} / \mathrm{cm}^{2}\right)$. Esta presión se seleccionó a partir de pruebas de porosidad obtenidas en aglomerados piroconsolidados. Todas las probetas fabricadas resultaron de un diámetro promedio de $8 \mathrm{~mm}$ y un espesor de $4 \mathrm{~mm}$, conteniendo alrededor del $25 \%$ de porosidad inicial.

\subsection{Sinterización}

Las probetas mencionadas se secaron en un horno de mufla a $100{ }^{\circ} \mathrm{C}$ durante $3 \mathrm{~h}$ para eliminar la humedad aportada durante el conformado y, posteriormente, se sinterizaron en aire en un horno tubular a $1.250,1.275,1.300$ y $1.325^{\circ} \mathrm{C}$, con un tiempo de permanencia a estas temperaturas de $10 \mathrm{~min}$, empleando el ciclo térmico que muestra la figura 1 .

\subsection{Ensayos de resistencia mecánica}

Las probetas sinterizadas se sometieron a ensayos de resistencia mecánica a compresión en frío en

TABLA II.- Composición química de las muestras preparadas

TABLE II.- Chemical composition of mixtures prepared

\begin{tabular}{|l|rrrrrrrr|}
\hline Probetas & Serp., \% & $\mathrm{Fe}_{\mathrm{T}}$ & $\mathrm{Al}_{2} \mathrm{O}_{3}$ & $\mathrm{SiO}_{2}$ & $\mathrm{MgO}$ & $\mathrm{CaO}$ & $\mathrm{S}$ \\
\hline \multirow{5}{*}{ Ácidas } & 0 & 65,69 & 0,750 & 3,210 & 0,38 & 1,470 & 0,0108 \\
& 1 & 65,63 & 0,757 & 3,570 & 0,76 & 1,450 & 0,0106 \\
& 2 & 64,40 & 0,764 & 3,930 & 1,13 & 1,440 & 0,0105 \\
& 3 & 63,77 & 0,771 & 4,280 & 1,49 & 1,430 & 0,0104 \\
& 4 & 63,16 & 0,778 & 4,680 & 1,85 & 1,420 & 0,0103 \\
& 5 & 62,56 & 0,785 & 4,960 & 2,20 & 1,410 & 0,0102 \\
\hline \multirow{5}{*}{ Básicas } & 0 & 64,16 & 0,732 & 3,135 & 0,37 & 3,762 & 0,0105 \\
& 1 & 63,27 & 0,736 & 3,450 & 0,74 & 4,145 & 0,0104 \\
& 2 & 63,00 & 0,747 & 3,800 & 1,10 & 4,560 & 0,0103 \\
& 3 & 62,74 & 0,759 & 4,120 & 1,47 & 4,950 & 0,0103 \\
& 4 & 62,49 & 0,769 & 4,500 & 1,83 & 5,400 & 0,0102 \\
& 5 & 62,25 & 0,780 & 4,800 & 2,19 & 5,760 & 0,0101 \\
\hline
\end{tabular}

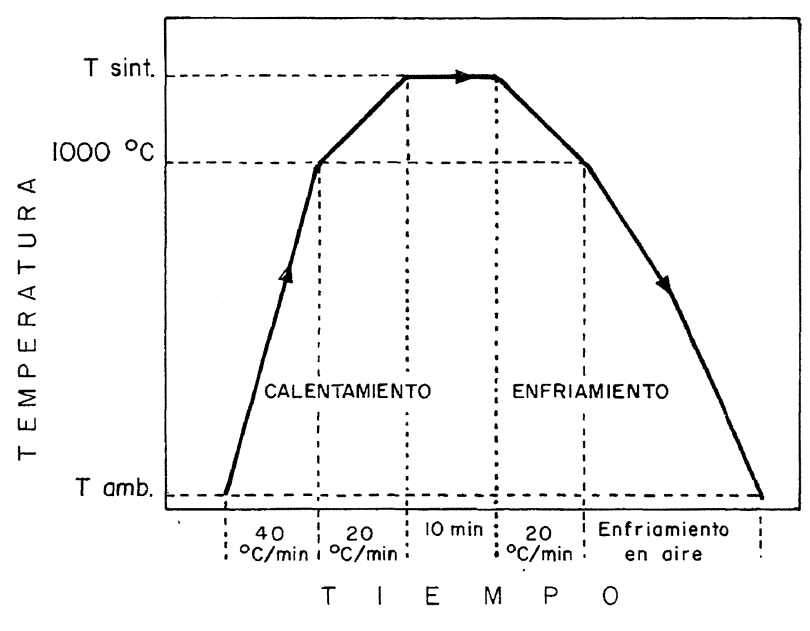

FIG. 1.-Ciclo térmico experimental.

Fig. 1.-Experimental firing cycle.

una máquina de pruebas universal INSTRON 1125 con registrador gráfico. El valor de la resistencia a la compresión, para cada una de las muestras, se determinó a partir de la media aritmética resultante de medir la resistencia de 20 probetas, en las cuales la carga se aplicó en dirección paralela al diámetro de la pastilla, como muestra la figura 2.

\subsection{Análisis microestructural}

Las muestras sinterizadas se examinaron por microscopía óptica. Para ello, se prepararon secciones planas desbastando las probetas con papel de carburo de silicio del núm. 600, puliéndolas a continuación con pasta de diamante de $1 \mu \mathrm{m}$ y empleando etanol como lubricante.

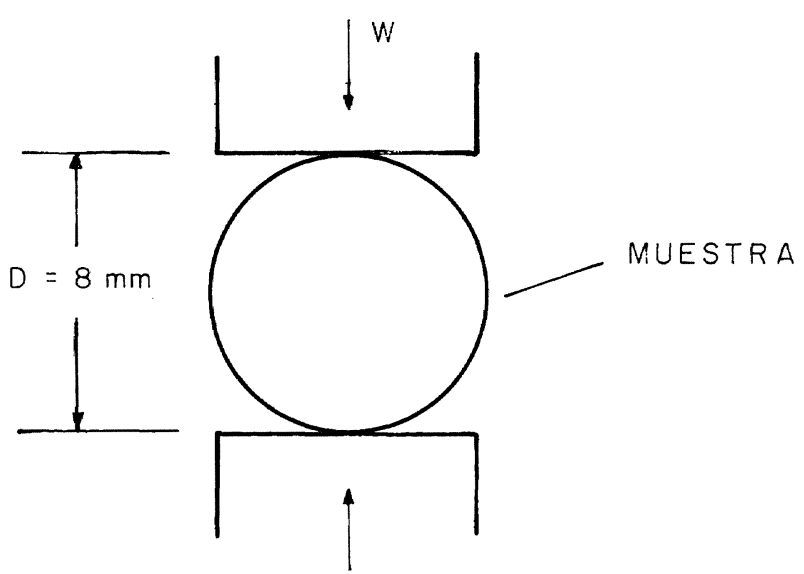

FIG. 2.- Representación esquemática de las pruebas de resistencia, donde $D$ es el diámetro de la pastilla y $W$ la carga aplicada $(\mathrm{kg})$.

Fig. 2.- Schematic illustration of mechanical test, where $\mathrm{D}$ is the diameter of the sample, and $\mathrm{W}$ is the breaking press load $(\mathrm{kg})$. 


\subsubsection{Identificación de fases}

Las fases mineralógicas presentes en las muestras sinterizadas se identificaron por difracción de rayos $\mathrm{X}$, microscopía óptica, microscopía electrónica de barrido y análisis por microsonda electrónica de barrido.

\subsubsection{Cuantificación de fases}

La cuantificación de fases en los sinterizados se estimó por examen óptico en un banco metalográfico LEITZ empleando el método de conteo por puntos, procedimiento que han usado con buenos resultados otros autores (7, 15 y 16). El método se fundamenta en la fracción del número total de puntos analizados que caen en la superficie correspondiente a una microestructura específica seleccionada (17). Se analizó un total de 500 puntos, resultado del barrido que se efectuó sobre la muestra al observar 10 campos visuales de manera horizontal y 10 en dirección vertical con 25 puntos cada uno. Dadas las dimensiones de las muestras, se considera que se efectuó un análisis representativo del contenido microestructural de las mismas. El valor porcentual en volumen de cada una de las fases (15) se calculó mediante la relación:

$$
\begin{gathered}
\text { Fase } \alpha, \%=\frac{P \alpha}{P_{\mathrm{T}}} \times 100 \\
\text { Porosidad, } \%=\frac{P_{\mathrm{P}}}{P_{\mathrm{T}}+P_{\mathrm{P}}} \times 100
\end{gathered}
$$

donde:

$P \alpha=$ Número de puntos correspondientes a la fase $\alpha$

$P_{\mathrm{P}}=$ Número de puntos de la fase porosa

$P_{\mathrm{T}}=$ Número total de puntos de todas las fases.

\section{RESULTADOS}

\subsection{Ensayos de resistencia mecánica}

La figura 3 muestra el efecto de la adición de serpentina sobre la resistencia mecánica en briquetas ácidas y básicas sinterizadas a diferentes temperaturas. De manera general, se observa que las probetas básicas presentan mayor resistencia que las ácidas en todo el rango de adición de serpentina estudiado. Además, queda claro que el incremento de la temperatura de sinterización induce a mayor resistencia mecánica en ambos casos.

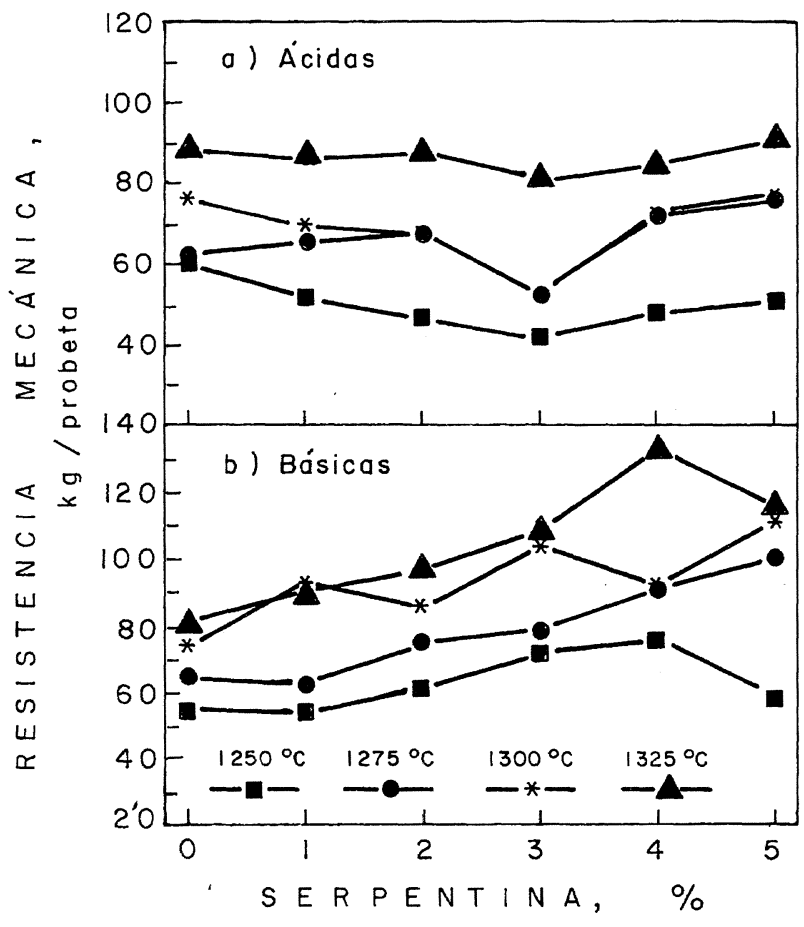

FIG. 3.- Efecto de la serpentina en la resistencia mecánica de las probetas sinterizadas a diferentes temperaturas: a) ácidas y b) básicas.

Fig. 3.- Effect of serpentine addition on mechanical strength of iron ore briquettes fired at several temperatures: a) acid briquettes and b) basic briquettes.

Las muestras ácidas presentan una caída de resistencia mecánica con el $3 \%$ de serpentina para todas las temperaturas, hecho que se atribuye principalmente a la presencia de magnesio-ferritas y escoria vítrea, así como a un mayor contenido de porosidad con respecto a las muestras que tienen distintos contenidos de serpentina.

En las probetas básicas, la adición de serpentina incrementa la resistencia mecánica como resultado de la aparición de calcio-ferritas y olivinas, además de la presencia de escorias que actúan como ligas intergranulares, y un bajo grado de porosidad. Las mejores propiedades de resistencia mecánica se obtuvieron con la pastilla básica $\left(\mathrm{CaO} / \mathrm{SiO}_{2}=1,2\right)$, con $4 \%$ de serpentina sinterizada a $1.325^{\circ} \mathrm{C}$.

\subsection{Observaciones estructurales}

En un examen detallado por microscopía de luz reflejada, se observaron las diferentes fases mineralógicas que exhiben los sinterizados de acuerdo con su composición química y con las condiciones de temperatura presentes durante el proceso de sinterización. Se encontró que la microestructura formada depende, principalmente, de la basicidad, del valor 
porcentual de serpentina y de la temperatura de sinterización.

Además de la relación que existe entre las microestructuras encontradas y las propiedades de resistencia mecánica que presenta cada una de las probetas ensayadas, se concluye que otro factor importante a considerar es la morfología que presentan dichas estructuras. De lo anterior, se puede deducir la importancia de realizar un examen mineralógico completo. La tabla III muestra las fases predominantes en los sinterizados en función de la basicidad y de la temperatura. Dichas fases se forman en diferente proporción con cada uno de los valores porcentuales de serpentina estudiados. Respecto a esta tabla, la fase escoria se refiere a aquellas escorias finas que actúan como ligantes entre granos.

A $1.250{ }^{\circ} \mathrm{C}$, las probetas ácidas sin aditivo presentan granos de sílice sin reaccionar comparables en tamaño con los cristales de hematites de tamaño medio (Fig. 4a). Existe la presencia de silicatos inmersos en la fase hematites, que se presenta en parte como cristales de bordes agudos generalmente aislados en matriz de escoria vítrea, o bien como granos diseminados en la escoria con presencia de porosidad intracristalina. En cuanto a las probetas básicas (Fig. 5a), la cantidad de sílice sin reaccionar disminuye considerablemente, la hematites presenta porosidad intracristalina con inclusiones mayores de escoria y espacios porosos intergranulares, además de la presencia de calcio-ferritas. Conforme se incrementa el contenido de serpentina aumenta la cantidad de sílice sin reaccionar en las probetas ácidas, considerándose ésta como un producto de la descomposición de la serpentina. La porosidad de las probetas también se ve incrementada y los granos de hematites toman una forma rugosa irregular con porosidad intracristalina poco menos marcada, donde los granos tienden a aglomerarse, aunque en forma quebradiza. En las briquetas básicas el incremento de la adición de serpentina origina una mejor distribución de la porosidad y mayor formación de

TABLA III.- Fases características en función de la basicidad y de la temperatura de sinterización

TABLE III.- Characteristic phases in function of basicity and firing temperature

\begin{tabular}{|c|cc|}
\hline Temp., ${ }^{\circ} \mathrm{C}$ & $\left(\mathrm{CaO} / \mathrm{SiO}_{2}\right)=0,3$ & $\left(\mathrm{CaO} / \mathrm{SiO}_{2}\right)=1,2$ \\
\hline 1.250 & $\mathrm{H}, \mathrm{S}, \mathrm{MF}, \mathrm{E}, \mathrm{P}$ & $\mathrm{H}, \mathrm{CF}, \mathrm{E}, \mathrm{P}$ \\
1.275 & $\mathrm{H}, \mathrm{S}, \mathrm{MF}, \mathrm{E}, \mathrm{P}$ & $\mathrm{H}, \mathrm{CF}, \mathrm{E}, \mathrm{P}$ \\
1.300 & $\mathrm{H}, \mathrm{S}, \mathrm{MF}, \mathrm{E}, \mathrm{P}$ & $\mathrm{H}, \mathrm{M}, \mathrm{E}, \mathrm{P}$ \\
1.325 & $\mathrm{H}, \mathrm{S}, \mathrm{MF}, \mathrm{E}, \mathrm{P}$ & $\mathrm{H}, \mathrm{M}, \mathrm{E}, \mathrm{P}$ \\
\hline
\end{tabular}

$\mathrm{H}$, hematites; $\mathrm{M}$, magnetita; $\mathrm{CF}$, calcio-ferrita; $\mathrm{MF}$, magnesio-ferrita $\mathrm{P}$, poros; $\mathrm{E}$, escoria; $\mathrm{S}$, sílice sin reaccionar. escorias finas intergranulares, con cristales compuestos de hematites más grandes y regulares.

$\mathrm{Al}$ incrementar la temperatura a $1.275^{\circ} \mathrm{C}$, en las muestras ácidas (Fig. 4b), los granos de hematites son más largos, densos y compactos, disminuyendo la porosidad intracristalina y la cantidad de sílice remanente. Los cristales siguen siendo irregulares, pero de bordes menos abruptos y se encuentran inmersos en escoria vítrea. La porosidad en las probetas básicas (Fig. 5b) muestra apariencia semiesférica de forma intergranular, presentando incrustaciones mayores de escoria en los cristales de hematites conforme se incrementa la adición de serpentina, de manera tal que ligan a los cristales de hematites con las calcio-ferritas que, por lo general, se presentan como pequeños granos rugosos perfectamente unidos por escorias o bien parcialmente diseminadas en la fase hematites.

A $1.300^{\circ} \mathrm{C}$, aparece la fase magnetita en briquetas básicas (Fig. 5c). La magnetita se manifiesta distribuida irregularmente, ligada en forma de granos individuales por escorias finas, o bien, en forma parcialmente diseminada en la fase hematites. Ambos tipos de probetas presentan porosidad intracristalina, principalmente las muestras ácidas, en las cuales se manifiesta una pequeña cantidad de hematites desarrollada de forma laminar en grandes zonas ricas en cristales de magnesio-ferritas que presentan ligeras inclusiones de escorias (Fig. 4c). Las magnesio-ferritas se incrementan con el contenido de serpentina y se muestran como granos pequeños inmersos en escoria vítrea, así como en forma de granos mayores de formas irregulares.

En condiciones básicas, a la temperatura máxima de sinterización de $1.325^{\circ} \mathrm{C}$, los granos individuales de la fase hematites tienen bordes ovalados ligados por escorias finas entre los mismos o con cantidades pequeñas de magnetita, presentando una ligera porosidad intracristalina (Fig. 5d). La porosidad intragranular es esférica y semiesférica, distribuyéndose regularmente en toda la muestra y formándose en algunos casos poros esféricos concéntricos a la fase magnetita. Las probetas ácidas presentan granos no muy grandes pero mejor definidos que a bajas temperaturas, con poca sílice sin reaccionar. La porosidad tiende a concentrarse y la cantidad de magnesio-ferritas disminuye de manera considerable, manifestándose la presencia de hematites de forma laminar (Fig. 4d).

\section{DISCUSIÓN}

Como se ha indicado, los factores más importantes que influyen en la resistencia mecánica de los sinterizados son la basicidad y el contenido de serpentina. La resistencia mecánica se ve favorecida con el 

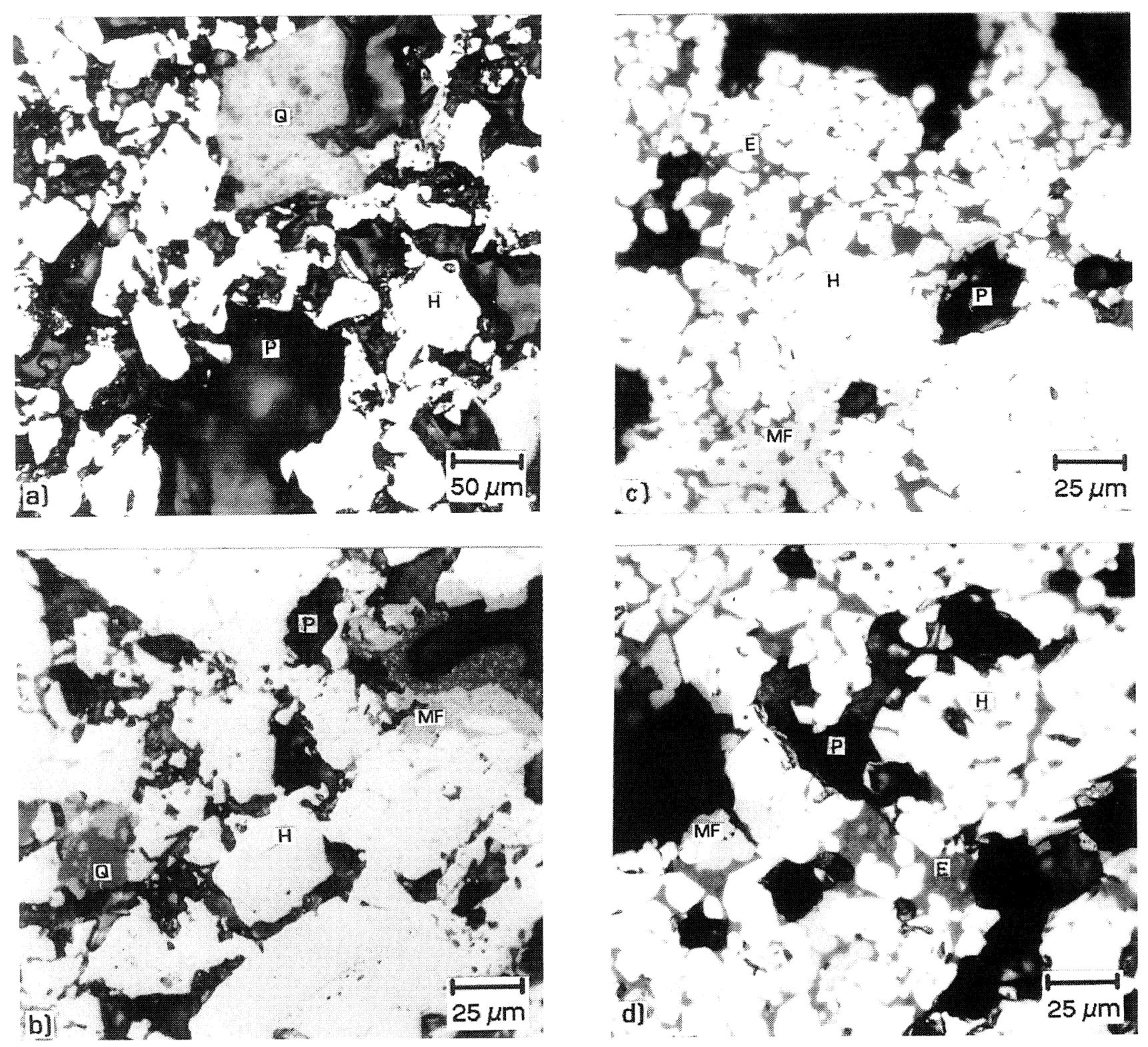

FIG. 4.- Micrografías de las probetas ácidas sinterizadas: a) $1.250{ }^{\circ} \mathrm{C}, 0 \%, \times 200$; b) $1.275{ }^{\circ} \mathrm{C}, 3 \%, \times 400$; c) $1.300{ }^{\circ} \mathrm{C}, 3 \%, \times 400$; y d) $1.325^{\circ} \mathrm{C}, 3 \%, \times 400$. H, hematites; P, poros; Q, sílice sin reaccionar; MF, magnesio-ferrita; E, escoria.

Fig. 4.- Micrographs of acid briquettes sintered at a) $\left.1,250{ }^{\circ} \mathrm{C}, 0 \%, \times 200 ; b\right) 1,275{ }^{\circ} \mathrm{C}, 3 \%, \times 400$; c) $1,300{ }^{\circ} \mathrm{C}, 3 \%, \times 400$; and d) $1,325^{\circ} \mathrm{C}, 3 \%, \times 400 . \mathrm{H}$, hematite; $P$, pore; $Q$, unreacted silica; $M F$, magnesioferrite; E, slag.

incremento de la temperatura, con la relación $\mathrm{CaO} / \mathrm{SiO}_{2}$ y con el contenido de $\mathrm{MgO}$. La resistencia del sinterizado depende, en gran medida, de las propiedades y cantidad de la matriz resultante, así como del resto de fases presentes que dependen en parte de la temperatura de sinterización de las muestras.

\subsection{Temperatura de $1.250{ }^{\circ} \mathrm{C}$}

\subsection{1. $\mathrm{CaO} / \mathrm{SiO}_{2}=0,3$}

Para probetas ácidas sinterizadas a $1.250{ }^{\circ} \mathrm{C}$, se obtienen los valores más bajos de resistencia mecá- nica, donde el valor mínimo corresponde al contenido de $3 \%$ de serpentina. Se observa que al aumentar la temperatura aumenta la proporción de hematites formada y, por tanto, es más elevada la resistencia mecánica (Figs. 3a) y 6a). El incremento de la resistencia se atribuye a que la hematites presenta la mayor microdureza entre las fases presentes $(1.000 \mathrm{HV})$.

La adición de serpentina induce la formación de magnesio-ferrita a todas las temperaturas, y esta fase se encuentra distribuida en toda la muestra unida a los óxidos a través de escorias. La magnesio-ferrita tiene una estructura de tipo espinela 

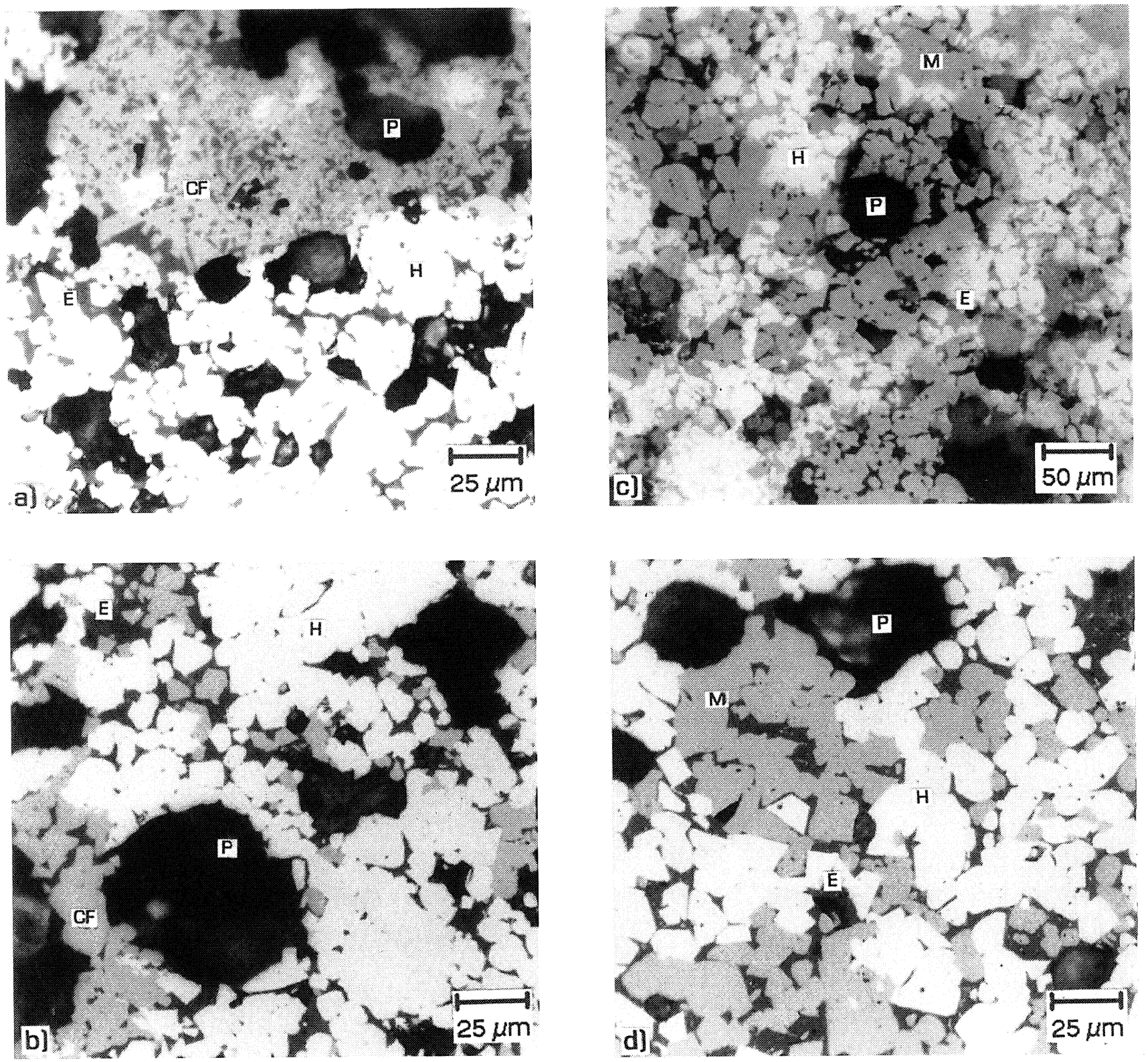

FIG. 5.- Micrografías de probetas básicas sinterizadas: a) $1.250{ }^{\circ} \mathrm{C}, 0 \%, \times 400$; b) $1.275{ }^{\circ} \mathrm{C}, 5 \%, \times 400$; c) $1.300{ }^{\circ} \mathrm{C}, 4 \%, \times 200$; y d) $1.325{ }^{\circ} \mathrm{C}, 4 \%, \times 400$. H, hematites; P, poros; $\mathrm{CF}$, calcio-ferrita; $\mathrm{M}$, magnetita; E, escoria.

Fig. 5.- Micrographs of basic briquettes sintered at a) $\left.1,250{ }^{\circ} \mathrm{C}, 0 \%, \times 400 ; b\right) 1,275{ }^{\circ} \mathrm{C}, 5 \%, \times 400$; c) $1,300{ }^{\circ} \mathrm{C}, 4 \%, \times 200$; and d) $1,325{ }^{\circ} \mathrm{C}, 4 \%, \times 400$. $\mathrm{H}$, hematite; $P$, pore; $\mathrm{CF}$, calcio-ferrite; $\mathrm{M}$, magnetite; E, slag.

inversa que se forma cuando el $\mathrm{Mg}^{2+}$ es transportado por las escorias líquidas, desplazando una fracción de los iones $\mathrm{Fe}^{2+}$ de la red de la magnetita para formar espinelas de tipo $(\mathrm{Fe}, \mathrm{Mg}) \mathrm{O}$. $\mathrm{Fe}_{2} \mathrm{O}_{3}$, hecho que han confirmado otros autores (14 y 18). La estructura de tipo espinela resultante supone un descenso de la resistencia mecánica. Conforme se incrementa la adición de serpentina, aumenta también la formación de magnesio-ferrita, según muestra la figura $6 \mathrm{~d}$ ). El punto correspon- diente al $3 \%$ presenta la mayor formación de magnesio-ferritas $\mathrm{y}$, por tanto, la más baja resistencia.

Ante la pequeña cantidad de $\mathrm{CaO}$ y la baja temperatura de sinterización, la formación de fase líquida es relativamente limitada y, cuando precipitan los cristales primarios, no hay suficiente penetración de la escoria entre los granos, por lo que la ligazón entre éstos es despreciable. Como resultado, se presentan grandes lagunas de escoria vítrea de alta fragilidad, propiedad que, como ya se ha 


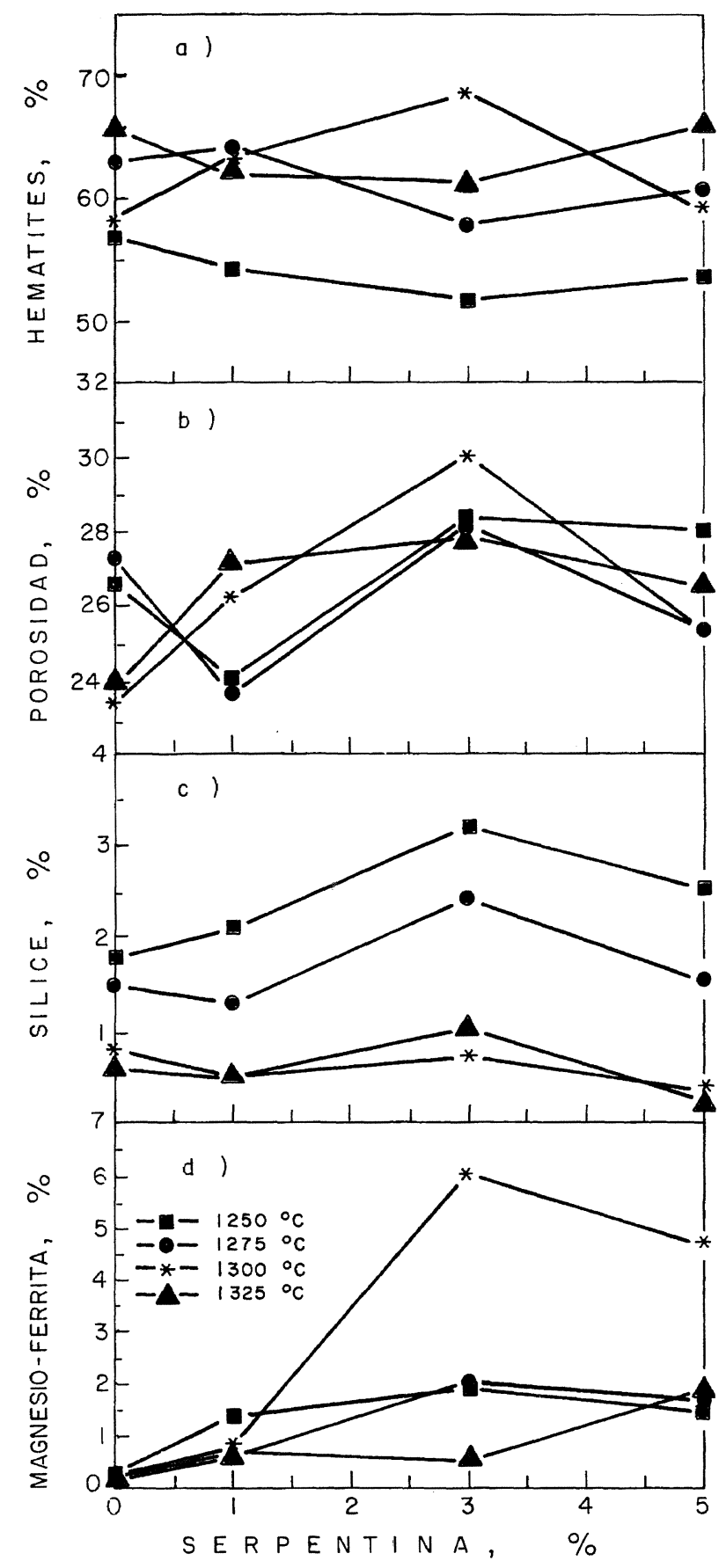

FIG. 6.- Influencia de la serpentina en la formación de: a) hematites, b) porosidad, c) sílice sin reaccionar y d) magnesio-ferrita, para probetas ácidas a las diferentes temperaturas de sinterización.

Fig. 6.- Influence of serpentine addition on mineralogical phases formation of iron ore acid briquettes fired at several temperatures: a) hematite, b) porosity, c) unreacted silica and d) magnesio-ferrite.

dicho, se atribuye a la concentración de esfuerzos propios de la presencia de grandes cantidades de escoria.
Un aspecto indicativo del bajo grado de sinterización a esta temperatura lo representa la sílice sin reaccionar (Fig. 6c). Por el comportamiento de las curvas de resistencia (Fig. 3a) se puede deducir que la sílice sin reaccionar constituye núcleos que disminuyen la resistencia de los sinterizados.

De la figura $6 b$ ) se deduce que la porosidad no muestra una tendencia clara a los cambios de temperatura, pero de manera general tiende a incrementarse con la adición de serpentina. El comportamiento de la porosidad es el mismo a temperaturas bajas de 1.250 y $1.275^{\circ} \mathrm{C}$, pero a las temperaturas altas de 1.300 y $1.325^{\circ} \mathrm{C}$ el comportamiento es distinto; sin embargo, para todas las temperaturas la máxima porosidad corresponde siempre a un mínimo de resistencia. Conforme se incrementa a más del $3 \%$ el contenido de serpentina, la porosidad disminuye ligeramente, lo que origina una recuperación de la resistencia mecánica.

\subsection{2. $\mathrm{CaO} / \mathrm{SiO}_{2}=1,2$}

En probetas básicas, la cantidad de hematites presente es menor en los puntos de máxima resistencia, siendo compensada esta disminución por la presencia de otras fases. A mayor temperatura de sinterización se eleva la cantidad de hematites, debido a que a temperaturas mayores aumenta la probabilidad de romper la refractariedad que proporciona el $\mathrm{MgO}$ al líquido y que puede evitar la precipitación de fases cristalinas (Fig. 7a).

A temperaturas bajas, de 1.250 y $1.275^{\circ} \mathrm{C}$, se forman calcio-ferritas (Fig. 7d), fase que se incrementa conforme se adicionan mayores cantidades de serpentina y cuya presencia mejora la resistencia mecánica de los sinterizados (Fig. 3b).

A $1.250{ }^{\circ} \mathrm{C}$, la adición de $\mathrm{MgO}$ a través de la serpentina incrementa ligeramente la porosidad debido a la baja producción de fase líquida. Para el caso de temperaturas elevadas disminuye la porosidad, hecho más notorio con el incremento de serpentina. Comparando las figuras $3 b$ ) y $7 b$ ), se advierte que al decrecer la cantidad de poros presentes en las muestras mejoran las propiedades de resistencia.

El carácter básico de las muestras aumenta la cantidad de fase líquida formada en el proceso de sinterización, por lo que hay más escorias que en las muestras ácidas. En general, la aparición de escorias se ve favorecida con el incremento de la temperatura y ligeramente con la adición de serpentina. Para todos los casos, la aparición de escorias finas intergranulares es proporcional al aumento o disminución de la resistencia, por lo que se deduce el efecto beneficioso de este tipo de escorias sobre 


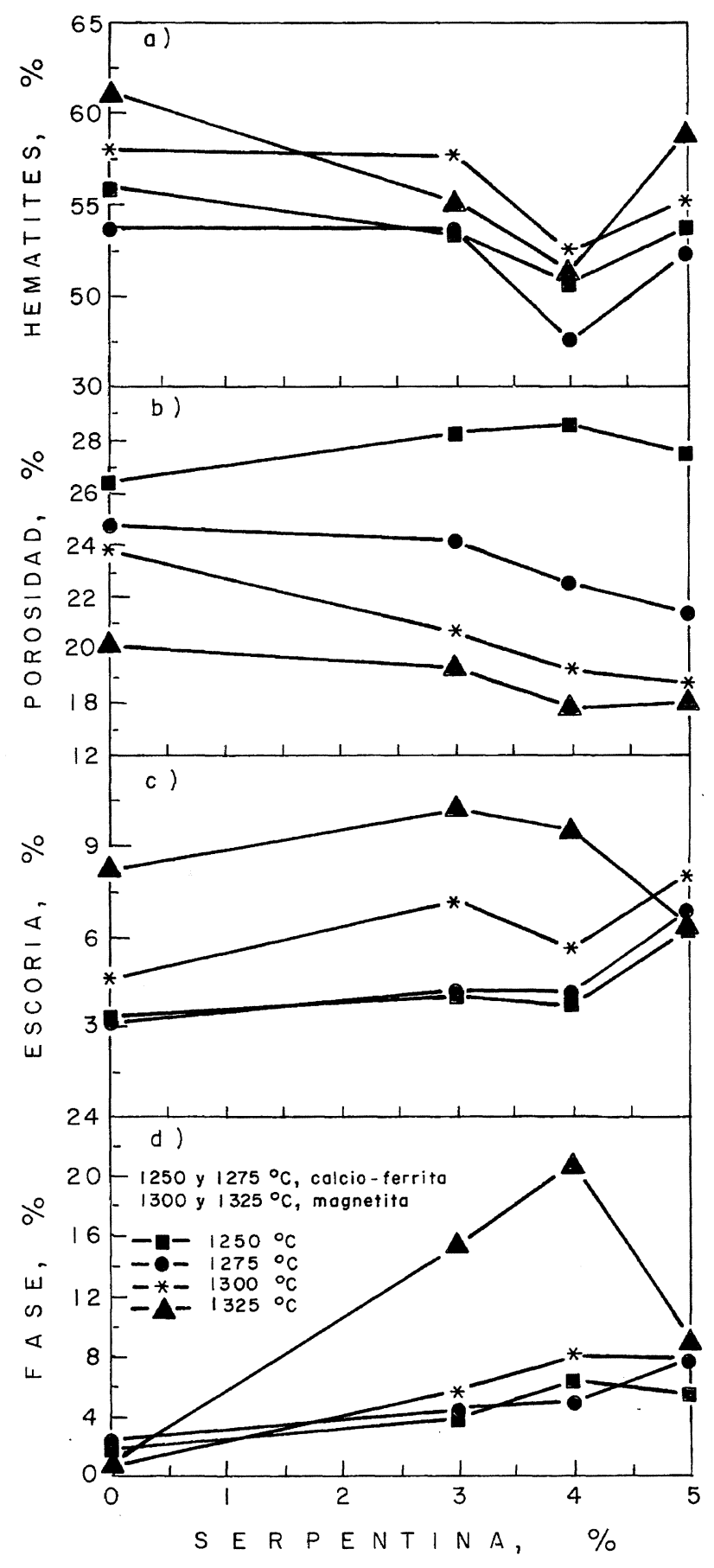

FIG. 7.- Influencia de la serpentina en la formación de: a) hematites, b) porosidad, c) escoria y d) calcio-ferrita y magnetita, para probetas básicas a las diferentes temperaturas de sinterización.

Fig. 7. Influence of serpentine addition on mineralogical phases formation of iron ore basic briquettes fired at several temperatures: a) hematite, b) porosity, c) slag and d) calcio-ferrite and magnetite. la resistencia mecánica en frío de los sinterizados (Figs. 3b) y 7c).

\subsection{Temperatura de $1.275^{\circ} \mathrm{C}$}

\subsection{1. $\mathrm{CaO} / \mathrm{SiO}_{2}=0,3$}

A 1.275 y $1.300{ }^{\circ} \mathrm{C}$, se observa una tendencia semejante de la resistencia mecánica. La presencia de hematites incrementa proporcionalmente la resistencia de las probetas, mientras que en el punto de mínima resistencia existe un aumento del contenido de magnesio-ferrita.

La escoria fina se encuentra más como inclusiones en los granos de hematites que en forma de ligante de los mismos y, en general, estos granos se encuentran inmersos en escorias vítreas que debilitan la estructura.

La presencia de sílice no asimilada, producto en parte de la descomposición de la serpentina, está relacionada con el incremento de la porosidad; así, por ejemplo, la muestra con $3 \%$ de serpentina presenta mayor cantidad de poros y sílice que el resto de las concentraciones.

\subsection{2. $\mathrm{CaO} / \mathrm{SiO}_{2}=1,2$}

En estas condiciones, la fase hematites resultó ser menor que con el resto de las temperaturas, disminuyendo ligeramente con el incremento de serpentina (Fig. 7a). El contenido de la calcio-ferrita formada es superior que a $1.250{ }^{\circ} \mathrm{C}$, puesto que se presenta mayor tiempo de contacto entre los productos sólidos iniciales que inducen a la formación de estas ferritas y que, en definitiva, permiten mayor disolución de los minerales de la ganga, generando así una cantidad superior de escorias. Este aumento del tiempo de contacto se debe a que conforme aumenta la temperatura de sinterización, las probetas se someten a un precalentamiento más prolongado que se extiende hasta lograr la temperatura máxima de quema.

A partir de estudios efectuados por microsonda electrónica de barrido, se detectó la presencia de silico-ferritas de calcio y alúmina, SFCA, que, como se sabe, contribuyen en gran medida a la resistencia de los sinterizados, y cuya formación tiene su origen en un efecto de estabilización de las calcio-ferritas con la alúmina presente en las muestras. En general, la presencia de calcio-ferritas supone una mejora de la resistencia mecánica, como se deduce de las figuras $3 \mathrm{~b}$ ) y $7 \mathrm{~d}$ ), pues promueve en ciertas regiones de los sinterizados, uniones entre los granos de hematites, que, aun cuando su presencia no es excesiva, se cree que tales estructuras pueden contrarrestar la propagación de 
grietas añadido ello al alto valor de dureza que pueden mostrar según el tipo de ferrita del que se trate y que varía de 800 a $900 \mathrm{HV}$, aproximadamente.

Con el incremento de la temperatura disminuye la porosidad. Se observa que en probetas ácidas la adición de serpentina produce siempre una mayor formación de poros. En condiciones básicas, la porosidad se ve incrementada ligeramente a 1.250 ${ }^{\circ} \mathrm{C}$, mientras que en el resto de las temperaturas, la porosidad disminuye conforme aumenta la relación $\mathrm{MgO} / \mathrm{CaO}$.

\subsection{Temperatura de $1.300{ }^{\circ} \mathrm{C}$}

\subsection{1. $\mathrm{CaO} / \mathrm{SiO}_{2}=0,3$}

A $1.300{ }^{\circ} \mathrm{C}$, se presenta un considerable descenso de la resistencia mecánica con un contenido de serpentina del $3 \%$ (Fig. 3a), donde la fase hematites se ve reducida en cantidad y es complementada por la aparición en gran medida de magnesio-ferrita (Fig. 6d). Con el incremento de la temperatura de sinterización se observa mayor formación de esta fase, que puede atribuirse a que el incremento de la temperatura de trabajo disminuye la viscosidad del líquido formado, favorece el transporte de los iones $\mathrm{Mg}^{2+}$ disueltos en la escoria hacia las estructuras magnetíticas e induce la formación masiva de magnesio-ferritas por el proceso descrito con anterioridad.

La disminución de la resistencia mecánica se atribuye, entonces, a la escasa dureza de la fase magnesio-ferrita (685 HV), sumada a los defectos estructurales que su presencia origina en los límites de grano que forma con la hematites y a la existencia de escorias vítreas distribuidas irregularmente a lo largo de la muestra.

Para el $5 \%$ de serpentina se presenta una recuperación de la resistencia, que se relaciona con la presencia de mayor cantidad de escorias finas intergranulares que ligan a los cristales de las distintas fases y a la disminución considerable de la porosidad (Fig. 6b).

\subsection{2. $\mathrm{CaO} / \mathrm{SiO}_{2}=1,2$}

A temperaturas elevadas, las ferritas dejan de ser estructuras estables, y la diferencia entre los sinterizados radica principalmente en la cantidad de magnetita observada en las muestras con alto contenido de serpentina. Se encontró que el incremento de la relación $\mathrm{MgO} / \mathrm{CaO}$ origina la reversión de hematites a magnetita. De acuerdo con lo señalado por otros autores (19), se concluye que la adición de $\mathrm{MgO}$ en las muestras estabiliza la estructura de tipo espinela, previniendo así una completa conversión a hematites durante el proceso de sinterización, con la consecuente presencia de magnetita a $1.300 \mathrm{y}$ $1.325^{\circ} \mathrm{C}$.

A las temperaturas de sinterización más elevadas, se presentan mejores escorias intergranulares, que contribuyen a mantener buenas ligas entre los granos mejorando las propiedades de resistencia de las probetas (Fig. 7c). Estudios por microsonda electrónica revelan que tales escorias contienen aluminio y magnesio, como resultado de la disolución de tales elementos en el líquido a alta temperatura.

\subsection{Temperatura de $1.325{ }^{\circ} \mathrm{C}$}

\subsection{1. $\mathrm{CaO} / \mathrm{SiO}_{2}=0,3$}

A esta temperatura se presenta mayor formación de hematites, hecho que se atribuye al mayor tiempo de precalentamiento transcurrido que permite la oxidación de $\mathrm{Fe}^{2+}$ a $\mathrm{Fe}^{3+}$, donde, de acuerdo con las figuras 3a) y 6a), se observa que los valores máximos de hematites corresponden a valores mayores de resistencia, constatando el efecto beneficioso de dicha fase sobre esta propiedad.

A $1.325^{\circ} \mathrm{C}$, la cantidad de magnesio-ferrita disminuye ligeramente. Como se ha mencionado, la magnesio-ferrita se forma principalmente por la sustitución de $\mathrm{Fe}^{2+}$ por $\mathrm{Mg}^{2+}$ de la estructura de la magnetita. También se ha indicado que conforme se incrementa el tiempo de precalentamiento decrece la cantidad de magnetita presente, debido a que en presencia de aire sufre una oxidación a hematites. A partir de lo anterior, se concluye que es menos probable la difusión de iones $\mathrm{Mg}^{2+}$ a la red de la escasa fase magnetita, por lo que la formación de magnesio-ferritas decrece cuando la temperatura es muy elevada (Fig. 6d).

La porosidad con el $3 \%$ de serpentina es menor que con el resto de las temperaturas, pero continúa siendo esta concentración de aditivo la que presenta un máximo de poros que produce, en unión con la sílice, menores valores de resistencia (Figs. 3a), 4b) y $4 c)$.

\subsection{2. $\mathrm{CaO} / \mathrm{SiO}_{2}=1,2$}

A estas condiciones de temperatura y basicidad se consigue la mejor resistencia mecánica de las probetas (Fig. 3b). En general, la porosidad tiende a disminuir conforme se incrementa la adición de serpentina. El punto que presenta menor contenido de poros es el correspondiente a $1.325{ }^{\circ} \mathrm{C}$ con $4 \%$ de serpentina, y es precisamente éste entre todos los sinterizados el que presenta la mejor resistencia.

La adición de serpentina da lugar a una mayor formación de magnetita por las mismas razones que 
a $1.300{ }^{\circ} \mathrm{C}$, y su aparición parece contribuir a la existencia de una porosidad más cerrada en las probetas, mejorando así las propiedades de resistencia (Fig. 7d). A temperaturas mayores, la escoria intercristalina fina se presenta en mayor cantidad incrementándose de manera general con la adición de serpentina a excepción de la muestra con $5 \%$, que presenta, además, una ligera caída de resistencia.

De acuerdo con otros estudios por microsonda electrónica, se detectó la presencia de olivinas de calcio, que resultan ser un producto de la desvitrificación de la matriz de escoria vítrea. Estas olivinas tienen una estructura continua y se encuentran depositadas principalmente en los límites de grano como parte de la escoria. Según han observado otros autores (14), la presencia de estructuras tales como olivinas y pyroxenos, productos de la desvitrificación, presentan una influencia favorable en la resistencia de los sinterizados.

\section{CONCLUSIONES}

- El incremento de la temperatura de sinterización mejora las propiedades de resistencia. Las probetas básicas muestran siempre mejor resistencia mecánica con respecto a las ácidas en el rango de temperatura estudiado.

- La adición de serpentina mejora la resistencia mecánica en las probetas básicas, mientras que en las ácidas, disminuye hasta el $3 \%$ para finalmente mostrar una ligera recuperación conforme se adiciona más serpentina.

- La resistencia mecánica en frío depende, en gran medida, de la mineralogía del sinterizado, que se relaciona con la temperatura, basicidad y valor porcentual de serpentina, principalmente.

- La adición de serpentina produce una mayor porosidad en las probetas ácidas a todas las temperaturas. En condiciones básicas, la porosidad se incrementa ligeramente a $1.250{ }^{\circ} \mathrm{C}$, mientras que a temperaturas mayores disminuye.

- En condiciones ácidas, el incremento de temperatura y la adición de serpentina promueven la formación de magnesio-ferritas, que debilitan el sinterizado por su escasa dureza y propagación de defectos estructurales. A $1.350{ }^{\circ} \mathrm{C}$ la magnesio-ferrita disminuye ligeramente, puesto que la cantidad de magnetita disminuye también por reoxidación a hematites.

- La resistencia en las probetas ácidas varía proporcionalmente con la fase hematites presente. En muestras básicas a temperaturas bajas, las calcio-ferritas contribuyen a mejorar la resistencia, mientras que, a mayor temperatura, con el incremento de la relación $\mathrm{MgO} / \mathrm{CaO}$, es más estable la magnetita, y en presencia de buenas escorias y olivinas mejora su resistencia.

\section{Agradecimiento}

Los autores agradecen a CONACYT y a la Universidad Michoacana de San Nicolás de Hidalgo el apoyo brindado para la realización de este trabajo.

\section{REFERENCIAS}

(1) Panigrahy, D.C., Verstraeten, P. y Dilewijns, J. Ironmaking Steelmaking, 10 (2), 1983: 54-64.

(2) Marliere, E., Steiler, J.M. y Jeulin, D. 5th Intern. Iron and Steel Congress. Washington D.C. 1986.

(3) Jeulin, D. Ironmaking Steelmaking, 10 (4), 1983: 145154.

(4) Cribbes, J.D. y Kestner, D.W. Agglomeration 77, 1, 1977: 272-285.

(5) Sastry, K.W.S., Negm, A. y Kater, T. 4th Intern. Symp. on Agglomeration. Toronto (Canadá), Jun., 1985.

(6) Ball, D.F., Darthell, J., Davison, J., Grieve, A. y WILD, R. Amer. Elsev. Publs. Comp., Inc. $1^{\underline{a}}$ Ed. Nueva York, 1963.

(7) Ball, D.F., Fitton, J.T., Dawson, P.R. y Goldring, D.C. Inst. Min. Metall. Mineral Process Extractive Metallurgy. Mar. 1974: 47-58.

(8) GuPTA, S. y Lu, W.K. Can. Metall. Quart., 26 (4), 1987: 329-339.

(9) Sugiyama, T., Shirouchi, S., Thuchiya, O., Onuda, M. y FuJiTA, I. Trans. ISIJ., 23, 1983: 153-160.

(10) Friel, J.J. y ERICKSON, E.S. Trans. Soc. Min. Eng. AIME., 273, 1980: 1.843-1.845.

(11) Sugiyama, T., Shirouchi, S., Tsuchiya, O., Onoda, M. y FuJITA, I. Trans. ISIJ., 23, 1983: 146-152.

(12) Panigrahy, S.C., Rigaud, M. y Dilewijns, J. Ironmaking Steelmaking, 11 (5), 1984: 246-252.

(13) Bedolla, E., Mendoza, G. y Lemus, J. ISIJ Intern., 34 (8), 1994: 631-640.

(14) Panigrahy, D.C., Verstraeten, P. y Dilewijns, J. Ironmaking Steelmaking, 11 (1), 1984: 17-22.

(15) HSIEH, L.-H. y Whiteman, J.A. ISIJ Intern., 33 (4), 1993: 462-473.

(16) Button, R.A. y Lundh, P.A. Ironmaking Steelmaking, 16 (3), 1989: 151-163.

(17) Underwood, E.E. Metallography and Microstructures. Metals Handbook 9. 1985: 123-134.

(18) Panigrahy, S.C. y Dilewijns, J. Ironmaking Steelmaking, 11 (2), 1984: 83-87.

(19) Friel, J.J. y ERickson, Jr., E.S. Metall. Trans. B, 11B, (2), 1980: 233-243. 